\title{
Special issue in quantitative mass spectrometric proteomics
}

\author{
Karin Barnouin
}

Received: 11 June 2012/ Accepted: 22 June 2012/Published online: 22 July 2012

(C) Springer-Verlag 2012

Proteomics research has increased exponentially since the 1980s upon the simultaneous and continuous development of biochemical purification and chromatography separation techniques, ionisation sources, fast scanning high mass accuracy mass spectrometers and bioinformatic tools. Completion of the human genome project in 2003 was instrumental in advancing research in this field by creating access to more complete protein sequence information.

In order for molecules to be analysed by mass spectrometry, they have to be ionised in an ionisation source prior to entering the mass spectrometer. In 2002, Koichi Tanaka and John Fenn jointly won the Nobel Prize in chemistry for the development of matrix-assisted laser desorption ionisation (MALDI) and electrospray ionisation (ESI) sources, respectively. In the 1980s Koichi Kanaka, Franz Hillencamp, and Michael Karas discovered that amino acids, proteins, and polypeptides could be ionised in a solid phase when mixed with a matrix and organic solvent, and laser ablated into a mass spectrometer (Karas et al. 1985; Tanaka et al. 1988; Hillenkamp et al. 1991; Beavis and Chait 1996). MALDI was particularly useful for the analysis of proteins subjected to endopeptidase digestion that generates peptides of specific mass, which can then be compared to a protein or genomic database to determine the identity of the protein (Yates et al. 1993; Cottrell 1994). This analysis is termed peptide mass finger printing (PMF).

\footnotetext{
K. Barnouin ( $\square)$

Protein Analysis and Proteomics Laboratory,

Clare Hall Laboratories, London Research Institute,

Cancer Research UK, Potters Bar,

Hertfordshire EN6 3LD, UK

e-mail: karin.barnouin@ cancer.org.uk
}

The concurrent development of electrospray ionisation (ESI) sources facilitated the analysis of biological macromolecules in liquid phase (Whitehouse et al. 1985). In this method molecules are ionised upon the application of a voltage to a capillary. In the charging process molecules are desolvated by electrospray dispersion and enter the mass spectrometer. ESI enabled the online analysis of molecules, including proteins and peptides, separated and purified by liquid chromatography. Development of nanoscale high performance liquid chromatography (nanoHPLC) meant that samples in the femtomole range could be analysed thereby significantly increasing analytical sensitivity. In addition, during the last decade major technological improvements of mass spectrometers have been made. In particular, the development of Thermofisher's hybrid linear ion trap/orbitrap mass spectrometers and new generation Q-TOFs in 2005 was a breakthrough. These instruments fast-scan and measure the mass of ions with a mass accuracy at sub part per million levels significantly increasing the amount of ions that could be analysed and the sensitivity of their detection. These improvements led to a more accurate analysis of complex samples. Today in mass spectrometry these analyses are performed not only on the MS level where accurate masses of peptides are measured, but also on the tandem MS (MS/MS) level where peptides or proteins are fragmented in the gas-phase into various lengths mainly between amino acid residues that enable the determination of their sequence from either the $\mathrm{N}$ or C-termini. The resulting masses from MS and MS/ MS fragment ions spectra are matched with a protein sequence database with bioinformatic search engines. With all these ground-breaking advances, most of today's biological mass spectrometrists work on developing applications while hardware improvements is now largely the realm of biotechnology companies. 
We are presently in a position to ask more complex biological questions related to the primary structure of proteins, especially post-translational modifications (PTM). These alterations in protein structure are caused by the additions or removal of chemical moieties that alter the tertiary structure and hence may modify biological regulation, activity and function of a protein. The most common and best understood modification is phosphorylation where this PTM increases the mass of a protein by $80 \mathrm{Da}$. It is thought that two-thirds of the human proteome is modified by phosphorylation of mainly serine, threonine or tyrosine amino acid residues (Mann et al. 2002). Phosphorylation has been best characterised in signal transduction pathways.

Besides phosphorylation other PTM's are also extremely important. For example, acetylation, sumoylation and ubiquitylation all modify lysines and have mostly been found to regulate protein stability or cellular localisation. There are many other PTMs but listing them here goes beyond the scope of this editorial. The majority of PTMs were previously identified by Edman degradation, immunochemical methods, amino acid analyses, and isotopic labelling (Larsen et al. 2006). Mass spectrometry has tremendously facilitated the identification of PTMs and has now become the analytical method of choice.

Nevertheless to answer complex biological questions, the elucidation of the identity of a protein and their PTMs is not enough. Biological processes are regulated by quantity or copy number and activity of a given protein. In addition, the proportion of a protein that is modified by PTMs usually represents a fraction of the total amount expressed in cells. The quantity expressed may influence the modulation of its activity and hence the function of the protein. Together with the technological improvements proteomics mass spectrometrists have devised chemical and biochemical methods to enrich and quantitate changes at both protein and PTM levels. These methods will be discussed further below.

In this Amino Acids special issue the articles were selected to reflect the progress and also common usage in quantitative proteomics. Several reviews address PTM and their quantitation from various angles ranging from method development to their applicability in biological questions. Some of the articles also present the potential issues researchers should be aware of when doing this type of analyses.

Two separate review articles by Fila and Honys (2012) and Beltran and Cutillas (2012) published here discuss the plethora of methods to enrich and detect phosphopeptides and proteins. The numerous methods that have been developed to facilitate the detection of phosphorylation indicate how difficult it may be to accurately identify and measure this seemingly abundant PTM. Although the identification of phosphorylation and their sites has become fairly routine, for each question there may not be one perfect method and several techniques may frequently be tested and applied in order to obtain a complete analysis.

Another fairly well characterised PTM is ubiquitylation of lysine. Ubiquitin itself is a small protein composed of 76 amino acids which can link to each other to form a chain. This chain can be of varying length and can be detected by SDS-page and Western blot as a ladder as it incrementally increases the mass of a poly-ubiquitylated protein. The presence of monoubiqutitylated proteins is less obvious. Ubiquitin is frequently identified in mass spectrometry by the detection of ubiquitin in the sample. The PTM site is identified after tryptic digest by a mass increase on lysine generated by a diGly tag that is appended to the peptide. Chen et al. (2012) in this issue review how ubiquitin PTM and other ubiquitin-like modifications such as SUMO can be detected and quantitated by mass spectrometry and how changes in their levels can be used to decipher their role in signalling pathways.

Once proteins and their PTMs have been identified, biologists, biochemists, and molecular biologists work on elucidating their function and regulation within the cell using quantitative proteomics methods. The most commonly applied quantitation methods are label-free and the isotopic labelling methods stable isotope labelling by amino acids in cell culture (SILAC, metabolic labelling) and isobaric tags for relative and absolute quantitation (ITRAQ, chemical labelling). In this issue Thompson et al. (2012) and Cappadona et al. (2012) give more details about these quantitation methods in terms of how they work and optimal mass spectrometric and bioinformatic analysis conditions.

Proteins and modifications do not act alone and also invariably affect the function and regulation of other proteins. There is cross-talk between signalling pathways. These observations led to the development of the field of study called systems biology. Since year 2000, biochemists, genomics and proteomics experts work together to study how gene regulation and protein expression and activity are interlinked and control signal transduction systems. In this issue, Jørgensen and Locard-Paulet (2012) discuss how qualitative and quantitative mass spectrometry can be used to analyse the cellular as well as the extracellular proteome and how these measurements can be used to determine phenotypic changes in disease.

Historically, quantitative analyses have been performed on the protein level which ultimately would be the most accurate way to evaluate how much of a protein is expressed in certain conditions at a given point in time. In this issue, Collier and Muddiman (2012) review the transition from quantitating intact proteins by $2 \mathrm{D}$ gel electrophoresis, especially differential gel electrophoresis to top- 
down mass spectrometric analyses. In the latter method, intact proteins with or without metabolic labelling are analysed directly (i.e. without previous proteolytic digestion), with or without prior HPLC purification by mass spectrometry. The proteins are in turn fragmented in the gas phase for identification purposes. The best data generated for these analyses are obtained by Fourier transform mass spectrometers. The generation of orbitrap instruments rendered this type of analyses more accessible. However, the data obtained from these analyses are extremely complex to analyse and more adequate and accessible software to interpret the MS/MS fragmentation data is required.

Consequently, the vast majority of qualitative and quantitative analyses in proteomics are performed using the bottom-up approach where proteins are digested with an endopeptidase, typically trypsin, and the protein inferred from the sequence of the peptides obtained from the analyses. In this special issue, Thompson et al. (2012) discuss the issues in the acquisition and analysis of qualitative peptide-centric proteomics experiments and how they may affect quantitative analyses.

Good bioinformatic tools are key to accurate data interpretation. However, good and easy-to-use software are often lagging behind technological developments and slow down or prevent the desired analyses. In addition, software has to be continually updated for ease of use, and most importantly to give accurate results. That said, as qualitative and quantitative analyses are increasingly becoming utilised this gap in development is becoming smaller. In this special issue, Cappadona et al. (2012) review critical aspects in quantitative software that are either freely or commercially available. The aim of this article is to increase awareness of both software developers and users of the software, the potential issues that should be considered in order to obtain precise quantitative analyses.

Finally, Sessler et al. (2012) provide an example of how a combination of biochemical separation and purification methods, qualitative and quantitative LC-MS and software development facilitate the analysis of protein complexes and determination of protein-protein interaction. Here, the researchers have adapted blue-native page electrophoresis in order to purify, identify, quantitate, and determine protein stochiometry of the proteins present in the proteosome complex of the malaria parasite Plasmodium falciparum. Without the continuous development of these complimentary analytical techniques as well as generation of gene and protein databases of all animal species these studies would not be possible.

As a final note I would like to thank all the authors for their excellent contributions for this special issue and their patience in the publication process. All articles have been externally peer reviewed and I thank the reviewers and also the production team for their invaluable support.

\section{References}

Beavis RC, Chait BT (1996) Matrix-assisted laser desorption ionization mass-spectrometry of proteins. Methods Enzymol 270:519-551

Beltran L, Cutillas PR (2012) Advances in phosphopeptide enrichment techniques for phosphoproteomics. Amino Acids (in this issue). doi:10.1007/s00726-012-1288-9

Cappadona S, Baker PR, Cutillas PR, Heck AJR, van Breukele B (2012) Current challenges in software solutions for mass spectrometry-based quantitative proteomics. Amino Acids (in this issue). doi:10.1007/s00726-012-1289-8

Chen P-C, Na CH, Peng J (2012) Quantitative proteomics to decipher ubiquitin signalling. Amino Acids (in this issue). doi:1007/ s00726-012-1286-y

Collier TS, Muddiman DC (2012) Analytical strategies for the global quantification of intact proteins. Amino Acids (in this issue). doi: 10.1007/s00726-012-1285-z

Cottrell JS (1994) Protein identification by peptide mass fingerprinting. Pept Res 7(3):115-124

Fila J, Honys D (2012) Enrichment techniques employed in phosphoproteomic. Amino Acids (in this issue). doi:10.1007/ s00726-011-1111-Z

Hillenkamp F, Karas M et al (1991) Matrix assisted laser desorption ionization mass spectrometry of biopolymers. Anal Chem 63:A1193-A1202

Jørgensen C, Locard-Paulet M (2012) Analysing signalling networks by mass spectrometry. Amino Acids (in this issue). doi: 10.1007/s00726-012-1293-z

Karas M, Bachmann D, Hillenkamp F (1985) Influence of the wavelength in high irradiance ultraviolet laser desorption mass spectrometry of organic molecules anal. Chem. Anal. Chem. 57:2935-2939

Larsen MR, Trelle MB, Thingholm TE, Jensen ON (2006) Analysis of posttranslational modifications of proteins by tandem mass spectrometry BioTechniques 40:790-798

Mann M, Ong SE, Grønborg M, Steen H, Jensen ON, Pandey A (2002) Analysis of protein phosphorylation using mass spectrometry: deciphering the phosphoproteome. Trends Biotechnol 20:261-268

Sessler N, Krug K, Nordheim A, Mordmüller B, Macek B (2012) Analysis of the Plasmodium falciparum proteasome using blue native PAGE and label-free quantitative mass spectrometry. Amino Acids (in this issue). doi:10.1007/s00726-012-1296-9

Tanaka K, Waki H, Ido Y, Akita S, Yoshida Y, Yoshida T (1988) Protein and polymer analyses up to $\mathrm{m} / \mathrm{z} 100000$ by laser ionization time-of flight mass spectrometry. Rapid Commun Mass Spectrom 2(20):151-153

Thompson AJ, Abu M, Hanger DP (2012) Key issues in the acquisition and analysis of qualitative and quantitative mass spectrometry data for peptide-centric proteomic experiments. Amino Acids (in this issue). doi:10.1007/s00726-012-1287-x

Whitehouse CM, Dreyer RN, Yamashita M, Fenn JB (1985) Electrospray interface for liquid chromatographs and mass spectrometers. Anal Chem 57(3):675-679

Yates JR 3rd, Speicher S, Griffin PR, Hunkapiller T (1993) Peptide mass maps: a highly informative approach to protein identification. Anal Biochem. 1;214(2):397-408 